\title{
Clinico-pathological studies of cattle naturally infected with Mycobacterium avium subspecies paratuberculosis in Khartoum State, Sudan
}

\author{
K B Mohammed ${ }^{*}$ and Zakia A. Mohamed \\ Department of Pathology and Diagnosis, \\ Central Veterinary Research Laboratories centre, P. O. Box 8067 (ALAmart), Khartoum, Sudan. \\ *Corresponding author email: khalidbabiker@hotmail.com \\ Received: 24-07-2011, Accepted: 12-09-2011, Published Online: 05-12-2011 \\ doi: $10.5455 /$ vetworld.2012.69-74
}

\begin{abstract}
Four crossbred cows, 3-5 year- old, naturally infected with Mycobacterium avium subspecies paratuberculosis (MAP) were sacrificed and necropsied. Clinically, they showed profused diarrhoea, emaciation and rough coat with an area of alopecia on the tail. The most prominent macroscopic lesions were thickening, oedema and corrugation of the wall of small and large intestines. The mesenteric lymph nodes were enlarged and oedematous. Microscopically, all cows presented granulomatous enteritis. The inflammatory exudates varied from accumulation of lymphoid cells mixed with some epithelioid macrophages and giant cells to sheets of epithelioid macrophages intermingled with some lymphoid cells. The lymphatics in the submucosa of both the small and large intestines were dilatated and filled with pink homogenous proteinous materials. Acid-fast bacilli (AFB) were demonstrated in the infiltrating epithelioid macrophages and giant cells. Culture of inocula from the small and large intestines and the mesenteric lymph nodes of all animals showed small, round, smooth and glistening colonies 5-7 weeks following incubation in Herrold's Egg Yolk Medium at $37^{\circ} \mathrm{C}$.
\end{abstract}

Keywords: Pathology, Cattle, Naturally, Mycobacterium paratuberculosis, Khartoum, Sudan

To cite this article : Mohammed KB and Mohamed ZA (2012): Clinicopathological studies of cattle naturally infected with Mycobacterium avium subspecies paratuberculosis in Khartoum State, Sudan, Vet. World 5 (2): 69-74, doi: 10.5455/vetworld.2012.69-74

\section{I ntroduction}

Paratuberculosis is a chronic enteropathy of ruminants caused by Mycobacterium avium subsp. paratuberculosis (MAP). Organisms ingested by neonatal animals are taken up by the Peyer's patches and infect intestinal macrophages. In most natural infections it is presumed that the host response eliminates the organisms, but some of the animals remain infected. Adult cows develop clinical disease following a long subclinical phase (Chiodini et al., 1984; Kreeger, 1991; Clarke, 1997).

Whitlock and Buergelt (1996) reported that the gross lesions of paratuberculosis were characterized by intestinal thickening and corrugation and the associated lymph nodes were swollen and oedematous. The main microscopic findings consist of granulomatous enteritis, lymphangitis and lymphadenitis associated with the presence of AFB in macrophages (Chiodini et al., 1984; Kreeger, 1991). Culture of intestinal tissues is more sensitive than culture of faecal materials. Animals negative in successive faecal cultures may become positive by culture from intestinal tissues after slaughter (Whittington and Sergeant, 2001). The aims of this study were to describe macrosopic and microscopic appearance of paratuberculosis in naturally clinical infected crossbred cows and to draw the correlation between impression smear and bacterial culture methods.

\section{Materials and Methods}

Pathological methods : Necropsies were carried out on four crossbred cows (No118, 119, 229 from Soba area, and 198 from Hilat Kuku area) that presented clinical signs of paratuberculosis. 


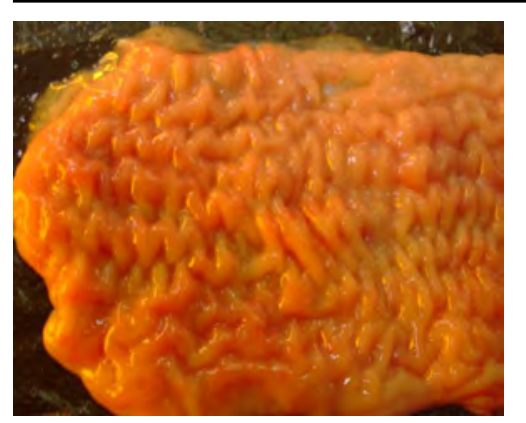

Fig 1: Ileum of animal No 229. Note, theFig mucosa was folded into thick transverse rugae.

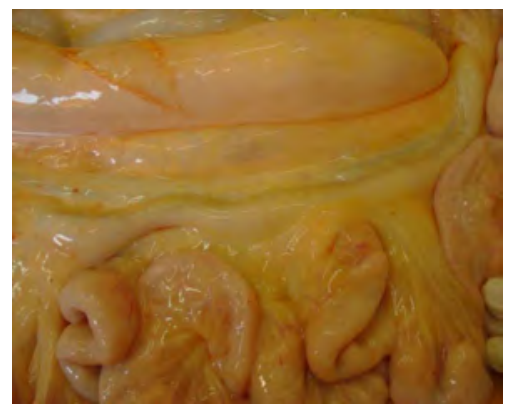

2: Note, swollen of mesenteric lymph node of animal No 198.

Fig 3: Jejunum of animal No 118. Note intense and diffuse infiltration of cells which efface the mucosal crypts.

(H\&EX10)
Specimens of the intestinal segments (duodenum, jejunum, ileum, caecum, colon and rectum), mesenteric lymph nodes, brain, heart, liver, kidneys and spleen were fixed in 10\% formalin and embedded in paraffin wax, sectioned at 4-5 $\mu \mathrm{m}$ thick and stained with haematoxylin and eosin $(\mathrm{H} \alpha \mathrm{E})$ and Ziehl-Neelsen $(\mathrm{ZN})$.

\section{Bacteriological methods}

I mpression smears : Impression smears were prepared from brains, hearts, kidneys, livers, spleens, mesenteric lymph nodes, large and small intestines of the four cows by impression of slides against a cut surface of each of the aforementioned and stained by Ziehl-Neelsen stain.

Preparation of inoculum : Approximately $4 \mathrm{~g}$ of mucosa from the specimens of intestinal segments (duodenum, jejunum, ileum, caecum, colon and rectum) and mesenteric lymph nodes were placed into a sterile blender jar containing $50 \mathrm{ml}$ of trypsin (2.5\%). The mixture was adjusted to neutrality ( $\mathrm{PH} 7$ ) using $4 \% \mathrm{NaOH}$ and stirred for $30 \mathrm{~min}$ at room temperature on a magnetic stirrer. The digested mixture was filtered through a gauze sheet. The filtrate was centrifuged at 2000-3000 g for $30 \mathrm{~min}$. The supernatant fluid was poured off and discarded. The sediment was resuspended in $20 \mathrm{ml}$ of $0.75 \%$ hexadecylpyridinium chloride (HPC) and allowed to stand undisturbed for 18 hours at room temperature. The particles that settled to the bottom of the tube were used as the inoculum and were removed by a pipette without disturbing the supernatant fluid.

Inoculation of culture media and incubation :
Approximately $0.1 \mathrm{ml}$ of inoculum was transferred to each of two slants of Herrold's medium containing mycobactin and to one slant of Herrold's medium without mycobactin. The innoculum was layered evenly over the surface of the slants. The tubes were allowed to remain in a slant position at $37^{\circ} \mathrm{C}$ for one week with the screw caps loose. The tubes were returned to a vertical position when the free moisture evaporated from the slants. The screw caps were tightened and the tubes were placed into baskets in an incubator at $37^{\circ} \mathrm{C}$ for 12 weeks and observed weekly from the sixth week onwards for the presence of MAP(OIE, 2004).

\section{Results}

Clinical signs: Though all four cows had good appetite, they were emaciated and had profused diarrhoea, rough coat with an area of alopecia on the tail. Mandibular oedema was observed in animal no 198.

Macroscopic findings : At necropsy, the animals were emaciated and showed serous atrophy of fat deposits. The most prominent gross lesions were thickening, oedema and corrugation of the wall of the small and large intestines. Mild pathological changes were observed in the duodenum and rectum. These changes were more marked in the lower jejunum and ileum, particularly at the ileocaecal valve where the mucosa was folded into transverse ridges that can not be reduced by stretching. Furthermore, animal no 118 showed segmented lesions with thickened portions of the small intestine being alternating with normal intestine. In the thickened portions, the mucosa 


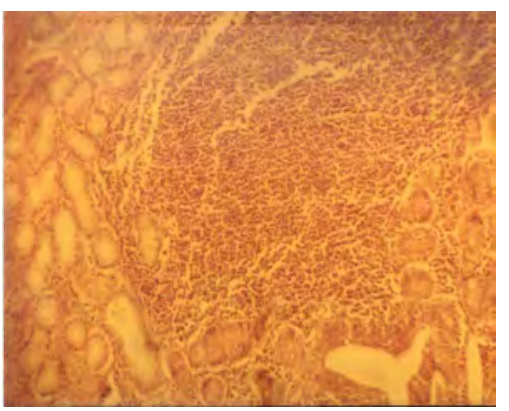

Fig 4: Duodenum of animal No 198 Note, diffuse infiltration of lymphoid cells.

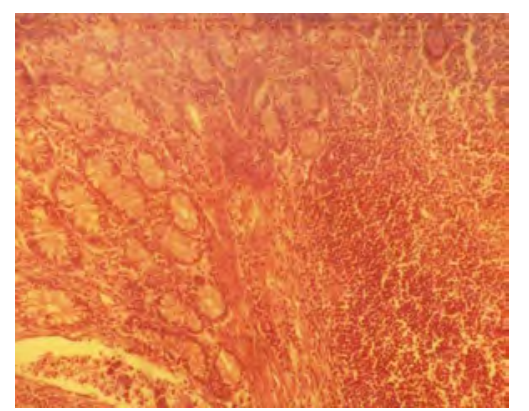

Fig 5: Jejunum of animal No 198. Note, Fig 6: Ileum of animal No 119. Note, intense and diffuse effusion of lymphoid clumps of acid-fast bacilli in the infiltrated cells and one langhans giant cell in the submucosa and mucosa. (H\&EX10).

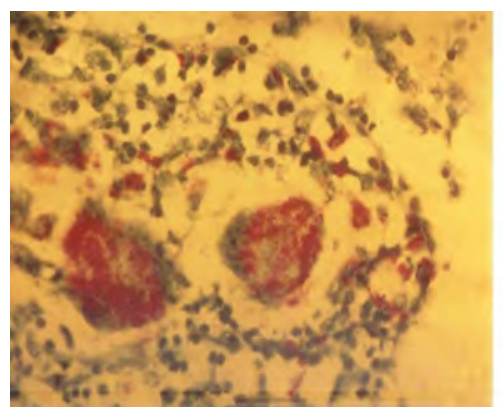
cells. (ZNX40) was folded into thick transverse rugae similar to the convolution of cerebral cortical gyrus (Fig.1).

Lymph nodes: The mesenteric lymph nodes were enlarged and oedematous especially in animals no 198 and 229 (Fig. 2). No other gross lesions were observed in the four emaciated cows, apart from congestion of the brain. Ecchymotic haemorrhages in the kidney was present only in animal no 198.

Microscopic findings : Generally, all animals presented granulomatous enteritis. Though, both the small and large intestines showed pathological changes, the severity of lesions varied considerably from moderate reaction in the proximal parts of the small intestine, duodenum, to an intense severe reaction in the distal part of the small intestine, lower jejunum and ileum, and mild changes in the large intestine.

The inflammatory exudates varied from accumulation of lymphoid cells mixed with some epithelioid macrophages and giant cells (Fig.3) to sheets or nests of epithelioid macrophages intermingled with some lymphoid and giant cells.

The lymphatics in the submucosa of both the small and large intestines were dilatated and filled with pink homogenous proteinous materials.

\section{Small intestine}

Duodenum : It showed mild villous atrophy with denudation of lining epithelium, moderate but diffuse infiltration of mononuclear cells, the majority of which were lymphoid cells, concomitant with some plasma and epithelioid macrophages in the mucosa, submucosa and between the compressed crypts of Lieberkuhn (Fig.4). Mild cellular reactions were observed between Brunner's glands and focal aggregates of epithelioid macrophages were encountered only at the tip of villi of animal no 198.

Jejunum and ileum : The most prominent changes were atrophic villi and desquamation of the lining epithelium. The changes were more marked in animal no 198 . The mucosal reaction was accompanied with intense and diffuse infiltration of mononuclear cells and few langhans giant cells in the mucosa and submucosa (Fig.5). However, the epithelioid macrophages effusion was prominent in animal no 118. In this animal the mucosa and submucosa were heavily infiltrated with nests or sheets of epithelioid macrophages resulting in compression and/or obliteration of mucosal crypts which in some areas were effaced by the inflammatory cells (Fig.3). In other parts the epithelioid macrophages form sheets either intermixed or encircled by mononuclear cell and few langhans giant cells. In animals no 119, 198 and 229 some mucosal glands or crypts were atrophic forming cyst-like structures filled with tissue debris. The submucosal reaction also varied from heavy infiltration of lymphoid cells to moderate or focal areas of lymphoid cells whereas in animal no 118 the predominant cellular reaction in the submucosa was sheets of epithelioid macrophages.

Acid-fast bacilli were encountered in the jejunum and ileum forming clumps in the infiltrating cells (Fig.6).

Large intestine : All segments of the large intestine showed mild villous atrophy, mild to 

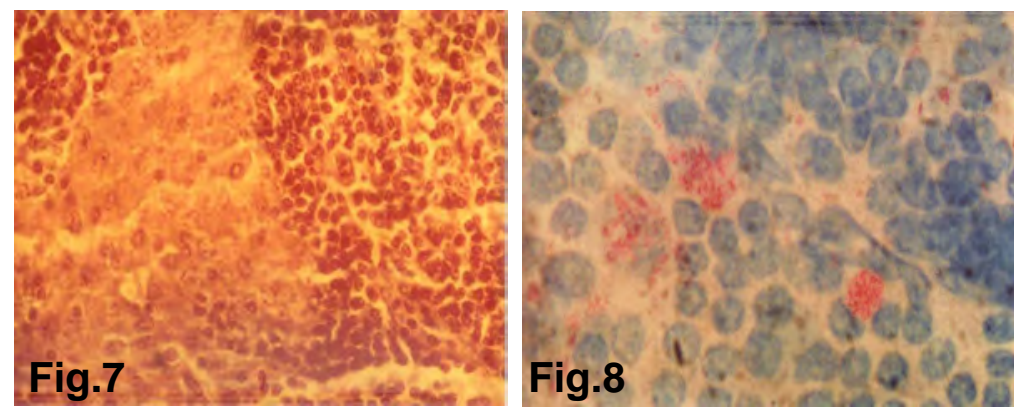

Fig 7: Lymph node of animal No 229. Note, sheet of epithelioid macrophages in the cortical area. H\&EX40.

Fig 8: Imprint of lymph node of animal No 198. Note, clumps of acid-fast bacilli intra and extracellular. ZNX40. moderate mononuclear reaction in the mucosa and submucosa. Focal collection of lymphoid cells were observed superficially or/and the base of the mucosa. However, animal no 118 showed sheets or nests of epithelioid macrophages in the mucosa and submucosa of the large intestine particularly the colon.

Lymph nodes: The mesenteric lymph nodes were enlarged, oedematous and hyperplastic with infiltration of mononuclear cells and sparse giant cells in subcapsular and medullary sinuses. The infiltrating cells were mainly lymphoid, some plasma and epithelioid macrophages. In animals no. 118 and 229, the epithelioid macrophages were found in nests throughout the lymph nodes (Fig.7). The medullary sinuses and lymphatics were filled with oedematous fluid. AFB were demonstrated in all sections and imprints of the mesenteric lymph nodes of all four examined cows (Fig.8).

Impression smears : Brains, hearts, kidneys, livers and spleens were negative AFB. The results of mesenteric lymph nodes, large and small intestines are summarized in Table- 1 .

Bacterial culture of organs : Decontaminated innocula prepared from tissue of small and large intestines and mesenteric lymph nodes incorporated in Herrold's egg yolk medium (HEYM) revealed colonies of MAP 5-7 weeks post incubation. These colonies were small, colourless, translucent and hemispherical. Their margins were round and even, whereas their surfaces were smooth and glistening (table 2).

\section{Discussion}

The clinical signs and macro-and microscopic findings observed in this study are similar to those described in MAP-infected cattle (Buergelt et al., 1978; Chiodini et al., 1984; Whitlock and Buergelt, 1996 Radostits et al., 2000).

In the present study emaciation and rough coat associated with alopecia were observed in all four crossbred cows. Mandibular oedema was only observed in animal no 198 which may be attributed to protein malabsorption and loss as a result of necrosis and destruction of intestinal mucosa of small intestine, particularly the ileum and jejunum where the ingesta are completely digested and absorption. This is similar to finding of Stehman (1990) and Radostits et al. (2000).

The profused diarrhoea observed presented by all four crossbred cows is the cardinal signs of paratuberculosis. The faeces were soft and thin, like thick pea soup, homogeneous and inoffensive. The faeces were neither blood nor mucous tinged. These findings were compatible with the

Table 1: Results of impression smears of large, small intestines and mesenteric lymph nodes from Crossbred cows in Khartoum State during 2006-2007.

\begin{tabular}{cccccccccc}
\hline Animal number & Source & \multicolumn{1}{c}{ Impression smear } \\
& & DU & J E & IE & ICV & CE & CO & RE & MLN \\
\hline 118 & Soba & - & + & + & ND & - & + & - \\
119 & Soba & - & + & + & + & + & + & + & + \\
198 & Kuku & - & + & + & + & + & - & + & + \\
229 & Soba & - & + & + & + & + & + & - & + \\
\hline
\end{tabular}


Clinico-pathological studies of cattle naturally infected with Mycobacterium avium subspecies paratuberculosis T

a b I e

\begin{tabular}{cccccccccc}
\hline Animal number & Source & \multicolumn{9}{c}{ Impression smear } \\
& & DU & JE & IE & ICV & CE & CO & RE & MLN \\
\hline 118 & Soba & + & + & + & ND & + & + & + & + \\
119 & Soba & + & + & + & + & + & + & - & + \\
198 & Kuku & - & + & + & + & - & - & - & - \\
229 & Soba & + & + & + & + & + & + & + & - \\
\hline
\end{tabular}

observations of other investigators (Buergelt et al.,1978; Whitlock and Buergelt, 1996). Moreover, in adult cattle the clinical disease should be differentiated from other diseases characterized by chronic diarrhoea. The chronic nature of paratuberculosis is usually sufficient to differentiate it from other common enteritis of cattle such as salmonellosis, coccidiosis and gastrointestinal helminthosis which are usually acute; the latter two disease occur principally in young animals and are distinguishable on faecal examination. Secondary copper deficiency (chronic molybdenum poisoning) is likely to be confused with paratuberculosis in cattle, but is usually a problem affecting large number of animals that responds well to the supplementation of copper (Radostits et al., 2000).

In the present study, the macro-and microscpic findings are similar to those described by others investigators (Jubb and Kennedy, 1970; Radostits et al., 2000). The severity of these changes in the lower small intestine and at ileocaecal valve may be attributed to the fact that these are predilection sites and the Peyer's patches are well developed and play an important role in mucosal immunity.

Serous atrophy of fat deposits, congestion of the brain in the four crossbred cows and ecchymotic haemorrhages in the kidneys that observed only in animal no 198. The first finding was observed previously (Buergelt et al., 1978). Congestion of brain and ecchymotic haemorrhages in the kidneys were not previously described in MAP-infected cattle.

Similar to the results of the the current investigation, the granulomatous inflammatory cells in the mucosa and submucosa of the small intestine and paracortex and sinuses of the mesenteric lymph nodes together with presence of intracellular AFB were reported by other investigators (Buergelt et al., 1978; Pembertson, 1979).

The mucosa and submucosa in animal no 118 were heavily infiltrated with nests or sheets of epithelioid macrophages resulting in compression and/or obliteration of mucosal crypts. This is in agreement with Radostits et al. (2000).

In the inflammatory exudation, in the four animals is comprised of mononuclear cells mainly lymphoid cells concomitant with granulation tissue. This indicate that the most important immuno mechanism operating in case of MAP-infected cows is cellular than humoral.

The mesenteric lymph nodes were enlarged, oedematous and hyperplastic with infiltration of mononuclear cells and sparse giant cells in the subcapsular and medullary sinuses. These cells were mainly lymphoid, some plasma and epithelioid macrophages. The medullary sinuses and lymphatics were distended with oedematous fluid. This is in agreement with the findings of Jubb and Kennedy (1970) and Radostits et al. (2000) but it is in contrast with the findings of Payne and Rankin (1961) who found that giant cells occurred less frequently in the intestines than in the associated lymph nodes.

Results presented in tables 1 and 2 indicate that there is poor correlation between impression smear and bacterial culture of organs for the detection of MAP in duodenum, caecum, rectum and mesenteric lymph nodes but it is high for detection of MAP in jejunum, ileum and ileocaecal valve. This indicates that the jejunum, ileum and ileocaecal valve are the predilection sites of choice for diagnosis of paratuberculosis in cattle for various tests (impression smear, bacterial culture and microscopic apperance). It is worth mentioning that, in view of the small 
number of animals studied, the observed differences in sensitivity of the various diagnostic methods must be evaluated carefully. The results do not give absolute proof that bacterial culture of organs is more sensitive than microscopic appearance. A large random scale study of local, crossbred lines and exotic cattle could elucidate those findings.

\section{Acknowledgements}

We gratefully acknowledge Prof. Osman, A.Y for critical reading of manuscript. This research was funded by The Central Veterinary Research Laboratories Centre, Khartoum, Sudan.

\section{Conflict of interest}

Authors declare that they have no conflict of interest

\section{References}

1. Buergelt, C. D.; Hall, C.; McEntee, K. and Duncan, J. R. (1978): Pathological evaluation of paratuberculosis in naturally infected cattle. Vet. Path., 15: 196-207.

2. Chiodini, R.J.; Van Kruiningen, H.J. and Merkal, R.S. (1984): Ruminant paratuberculosis (Johne's disease):the current status and future prospects. Cornell. Vet., 74:218-262.

3. Clarke, C.J. (1997): The pathology and pathogenesis of paratuberculosis in ruminants and other species. J. Comp. Pathol., 116:217.

4. Jubb, K.V.F. and Kennedy, P.C. (1970): Pathology of Domestic Animals, $2^{\text {nd }}$ Edit., Academic Press,
New York. pp. 135-140.

5. Kreeger, J. M. (1991). Ruminant paratuberculosisa century of progress and frustration. J. Vet. Diag. Invest ., 3:373-382.

6. Office International Des Epizooties. (2004): Manual of diagnostic tests and vaccines for terrestrial animals (mammals, birds and bees). $5^{\text {th }}$ edn. Paris, France. pp. 347-359.

7. Payne, J. M. and Rankin, J. D. (1961): The pathogenesis of experimental Johne's disease in calves. Res. Vet. Sci., 2: 167-174.

8. Pembertson, D.H. (1979): Diagnosis of Johne's disease in cattle using mesenteric lymph node biopsy: accuracy in clinical suspect. Aust. Vet. J., 55:217-219.

9. Radostits, O.M.; Gay, C.C.; Blood, D.C. and Hinchcliff, K.W. (2000): Veterinary Medicine, A textbook of the Diseases of Cattle, Sheep, Pigs, Goats and Horses. $9^{\text {th }}$ ed.Saunders London. pp. 920-934.

10. Stehman, S.M. (1990): Johne's disease (paratuberculosis). In: Smith, B. (Ed.), Large Animal Internal Medicine, The C.V. Mosby Co., St. Louis, pp. 823-829.

11. Whitlock, R.H. and Buergelt, C. (1996): Preclinical and clinical manifestations of paratuberculosis (including pathology). Vet. Clin. North Am. Food Anim. Pract., 12:345-356.

12. Whittington, R.J. and Sergeant, E.S. (2001): Progress towards understanding the spread, detection and control of Mycobacterium avium subsp. paratuberculosis in animal populations. Aust. Vet. J., 79: 267-278. 